\title{
Intoxicação natural por Solanum glaucophyllum (Solanaceae) em búfalos no Pantanal Matogrossense ${ }^{1}$
}

INDEX TERMS: Poisonous plants, Solanum glaucophyllum, Solanaceae, plant poisoning, buffaloes.

RESUMO.- Descreve-se a ocorrência de calcinose enzoótica em búfalos no município de Poconé, Mato Grosso, associado ao consumo de Solanum glaucophyllum ( $\mathrm{Sg}$ ) [=Solanum malacoxylon]. Os casos foram observados entre os anos de 2007 e 2009. Em um rebanho de 40 búfalos, cinco apresentaram emagrecimento progressivo, dorso arqueado, marcha rígida, por vezes com dificuldade para se levantar e locomover, permanecendo apoiando sobre os carpos.Três

\footnotetext{
${ }^{1}$ Recebido em 3 de agosto de 2011.

Aceito para publicação em 26 de agosto de 2011.

${ }^{2}$ Programa de Pós-Graduação e Medicina Veterinária, área de concentração Clínica Médica Veterinária, Universidade Estadual Paulista (Unesp), Jaboticabal, SP 14870-000, Brasil. *Autor para correspondência: Departamento de Clínica Médica Veterinária, Faculdade de Agronomia, Medicina Veterinária e Zootecnia, Universidade Federal de Mato Grosso (UFMT), Av, Fernando Correa da Costa 2367, Boa Esperança, Cuiabá, MT. 78068-900, Brasil. E-mail: carloseduardo@ufmt.br

${ }^{3}$ Departamento de Clínica Médica Veterinária, Faculdade de Agronomia, Medicina Veterinária e Zootecnia, UFMT, Boa Esperança, Cuiabá, MT.

${ }^{4}$ Empresa Matogrossense de Pesquisa, Assistência e Extensão Rural (Empaer), Rua Coronel Teófilo 600, Poconé, MT 78175-000, Brasil

${ }^{5}$ Departamento de Clínica e Cirurgia Veterinária, Faculdade de Ciências Agrárias e Veterinárias, Unesp, Jaboticabal, SP.
}

animais recuperaram-se parcialmente e dois foram eutanasiados in extremis. Os principais achados de necropsia foram calcificação de tecidos moles, principalmente em artérias de grande e médio calibres. A presença de S. glaucophyllum nas pastagens, os sinais clínicos, além dos achados ultrassonográficos e patológicos envolvendo múltiplas calcificações de tendões e outros tecidos, são compatíveis com intoxicação por Solanum glaucophyllum.

TERMOS DE INDEXAÇÃO: Plantas tóxicas, Solanum glaucophyllum, Solanaceae, intoxicação por planta, búfalos.

\section{INTRODUÇÃO}

Calcinose enzoótica, ocorre em vários países e recebe denominações como "enteque seco", na Argentina, "Manchester wasting disease", na Jamaica, "naahelu disease", no Havaí, "weidekrankhot", na Alemanha e Áustria, e "espichamento", no Brasil (Döbereiner et al. 1971). Esses quadros clínicos são relacionados a uma variabilidade de plantas que apresentam atividade calcinogênica, como Trisetum flavescens, Cestrum diurnum e Solanum torvum que acometem comumente bovinos (Dirksen et al. 1970, Copland 1975, Krook et al. 1975a,b). 
Acredita-se que a doença se deve à presença de glicosídeos com ações similares à vitamina $\mathrm{D}$ ativa, que causa hipercalcemia e hiperfosfatemia, com deposição excessiva de minerais em tecidos moles e calcificação de fibras elásticas (Mello 2003, Riet-Correa et al. 2007).

No Brasil, os quadros de calcinose ocorrem nos estados de Mato Grosso, Goiás, Mato Grosso do Sul e Rio Grande do Sul. Em Mato Grosso, calcinose em bovinos têm sido atribuídos à ingestão de Solanum glaucophyllum $(\mathrm{Sg})$ [=Solanum malacoxylon] e foram detalhadamente estudados natural e experimentalmente no bioma Pantanal (Döbereiner et al. 1971, Tokarnia \& Döbereiner 1974). No Rio Grande do Sul os quadros foram atribuídos à ingestão natural de Nierembergia veitchii (Riet-Correa et al. 1987, Barros et al. 1992, Riet-Correa et al. 1993, Rissi et al. 2007). No Brasil Central, em áreas de cerrado, casos naturais de calcinose foram assinalados, entretanto, a etiologia ainda é incerta; acometem bovinos e principalmente ovinos (Santos et al. 2005, Boabaid et al. 2006).

Este trabalho tem por objetivo, relatar aspectos epidemiológicos, clínicos e patológicos da calcinose enzoótica em bufalinos criados em condições extensivas no Pantanal Matogrossense.

\section{MATERIAL E MÉTODOS}

Dados epidemiológicos e sinais clínicos foram obtidos mediante visitas no período de 2007 a 2009, em propriedade localizada nas coordenadas $\mathrm{S}^{\circ} 6^{\circ} 21^{\prime} 53,5^{\prime \prime}$ e W 056 $41^{\prime} 07,0^{\prime \prime}$ com altitudes médias de $160 \mathrm{~m}$, por meio de inquéritos dirigidos aos tratadores e aos técnicos da assistência veterinária local. 0 georeferenciamento da área de ocorrência foi obtido com uso equipamento de GPS portátil com base no Datum SAD 69. Exames ultrassonográficos com escaner linear de $8.0 \mathrm{MHz}$ portátil foram realizados com varreduras nas áreas dos tendões flexores, região metacárpica, acima e logo abaixo dos sesamóides, utilizando-se, em secções longitudinais e transversais, respectivamente. Dois animais foram necropsiados e as amostras de pulmão, fígado, coração, rim, aorta, encéfalo foram obtidas e imediatamente fixadas em formalina $10 \%$ e processadas pela técnica padrão de LUNA (1968) e analisadas em microscopia óptica no Laboratório de Patologia Veterinária da UFMT.

\section{Epidemiologia}

\section{RESULTADOS}

A doença foi observada em um rebanho de quarenta búfalos nos anos de 2007 e 2009, na estação das chuvas, entre os meses de janeiro a março. No ano de 2007 constataram-se três búfalos apresentando sinais clínicos compatíveis com calcinose, em área infestada por Solanum glaucophyllum (Fig.1). A pastagem era diversificada em campos de pastos e plantas nativas em permeio a forrageiras de Brachiaria humidicola e B. decumbens. Após observação dos sinais de calcinose, os animais foram removidos da área. Em dois deles o quadro clínico se estabilizou e em outro se agravou. No ano de 2008 não se observaram novos casos, porém no final deste mesmo ano os animais foram novamente colocados na mesma área e o evento se repetiu em outros dois búfalos, com agravamento em um deles, em janeiro de 2009. Os casos concentraram-se em fêmeas adultas.

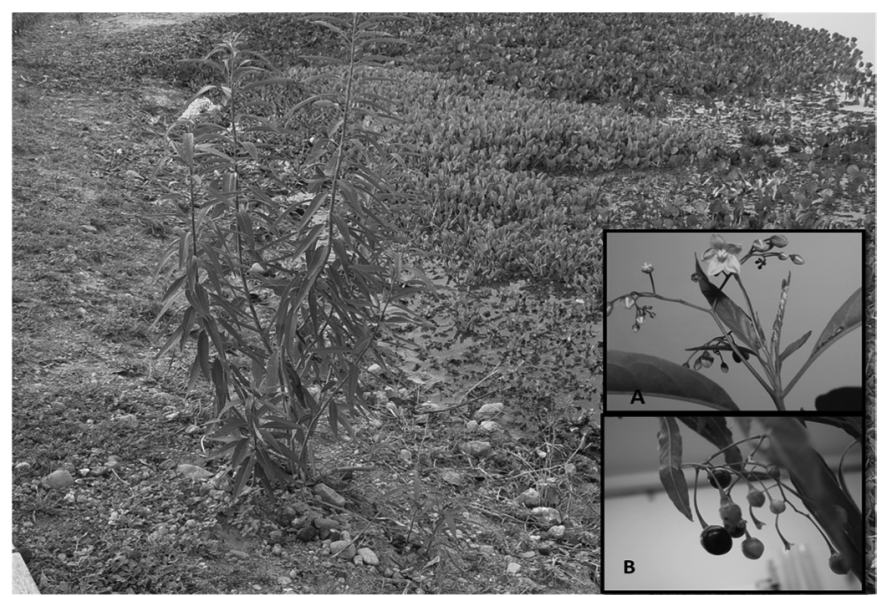

Fig.1. Solanum glaucophyllum (Sg) [=Solanum malacoxylon] em março de 2009, no Pantanal Norte, município de Poconé, MT, (A) com detalhe da inflorescência em março de 2007 e (B) frutificação em maio de 2009.

\section{Sinais clínicos}

Os búfalos exibiam caquexia, andar rígido com passos curtos, dorso arqueado, claudicação e apoio nas pinças dos cascos. Quando em estação, mantinham os membros torácicos ligeiramente flexionados (Fig.2). Por vezes, apresentavam dificuldades para se levantar, mantendo-se algum tempo ajoelhados ou mesmo pastando nesta posição (carpeados). Devido a esta postura, na articulação cárpica notou-se alopecia e hiperceratose circular na área de apoio. Também, os búfalos acometidos, gradativamente desenvolviam hipotricose. Os sinais vitais encontravam-se dentro dos parâmetros normais considerados para a espécie bufalina.

\section{Achados ultrassonográficos}

A extremidade distal dos membros torácicos foram avaliadas por meio de ultrassonografia com escaner linear de $8 \mathrm{Mhz}$, em secções longitudinais e transversais (Fig.3); em dois animais com sintomas da doença foram visibilizadas diversas calcificações focais caracterizadas por ninhos hiperecóicos nos tendões do músculo digital flexor profundo acima e abaixo dos sesamóides proximais.

Quadro 1. Demonstrativo da variação do escore corporal e calcificação de tecidos à necropsia em casos espontâneos da intoxicação por Solanum glaucophyllum em dois búfalos, fêmeas, SRD, adultas no município de Poconé-MT, nos anos de 2007 a 2009

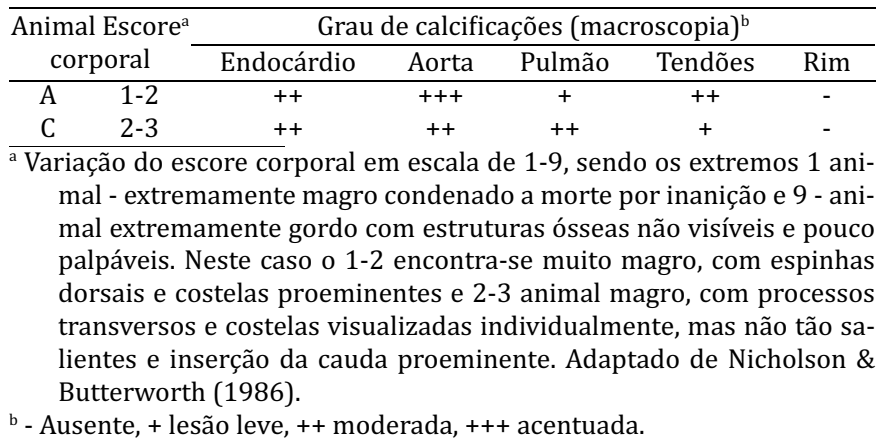




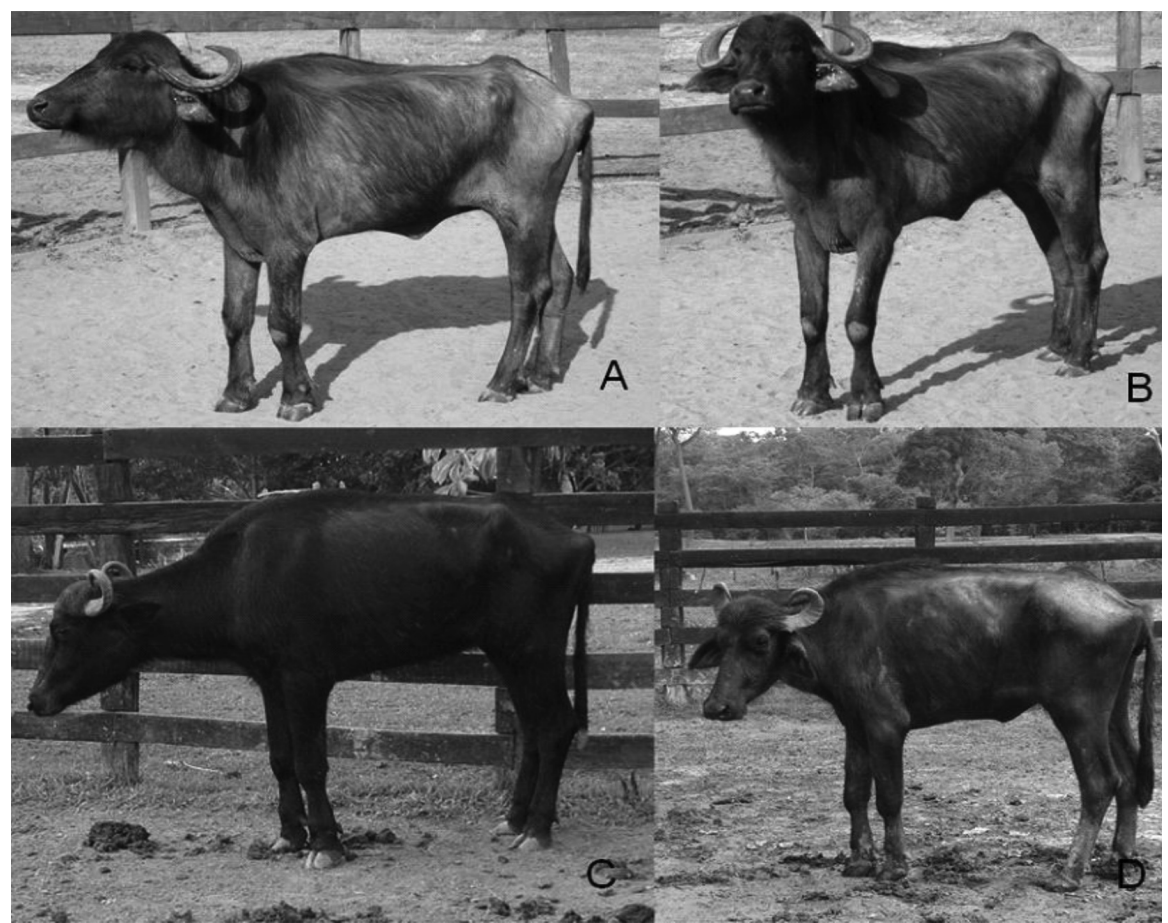

Fig.2. Búfalos exibindo sinais de hipotricose, caquexia, dorso arqueado e membros torácicos ligeiramente fletidos apoiando-se sobre as pinças, postura decorrente de intoxicação natural por Solanum glaucophyllum. (A,B) 0 mesmo animal em posições diferentes com alopecia e hiperceratose de apoio na região da articulação cárpica. (C,D) Outro búfalo com intervalo de quatro meses entre as imagens, demonstrando progressiva emaciação e perda de pêlos.

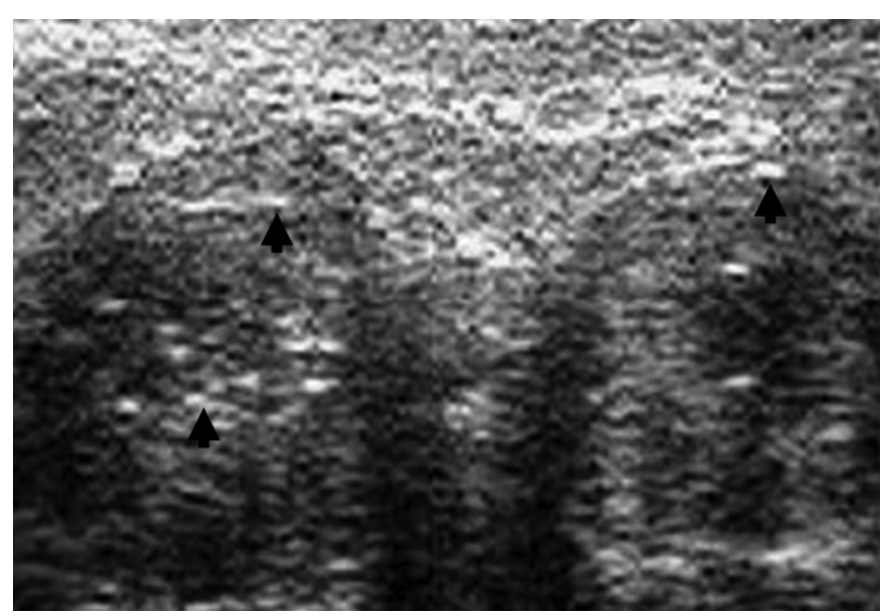

Fig.3. Imagem ultrassonográfica dos tendões do músculo flexor profundo imediatamente abaixo dos sesamóides, em plano transversal, obtida com transdutor linear de 8,0 MHz. Notar pontos hiperecóicos (setas) que correspondem a diversas calcificações.

\section{Achados patológicos}

À necropsia, os búfalos apresentavam-se caquéticos e com hipotricose generalizada. Os pulmões permaneciam armados e com aparente enfisema em distribuição aleatória, sendo observados alguns pontos com ossificação. Nas artérias havia irregularidade da superfície da íntima, por vezes recobertas com placas brancacentas principalmente aorta (Fig.4), carótidas e braquiais. No coração identificou-se calcificação, espessamento e opacidade de válvulas e cordas tendíneas que estavam mais consisten- tes e menos flexíveis que o normal. Algumas porções dos tendões flexores dos membros torácicos apresentavam pontos esbranquiçados acentuados na porção distal, próximo aos sesamóides. A síntese dos achados estão dispostas no Quadro 1.

Histologicamente as principais alterações foram nas artérias aorta e carótida que tinham formação de placas basofílicas, irregulares, multifocais por vezes coalescente principalmente na túnica média (Fig.5). Notava-se também desorganização e fragmentação das fibras na túnica média. Em um dos búfalos próximo às áreas de mineralização havia

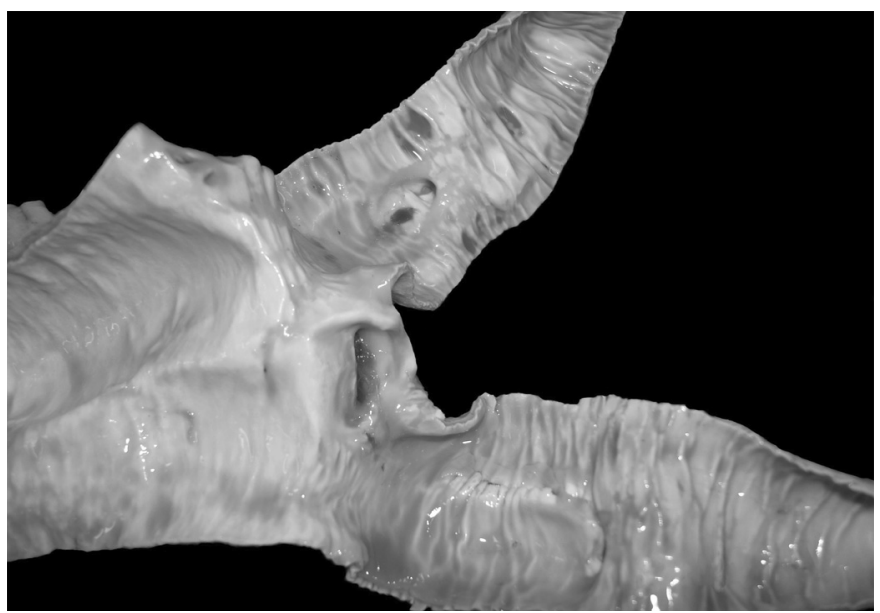

Fig.4. Artéria aorta e ilíacas de búfalo exibindo irregularidade da superfície intimal por processos de calcificação. Intoxicação natural por Solanum glaucophyllum. 
área com células semelhantes a condroblastos (metaplasia cartilaginosa). Frequentemente se notava proliferação multifocal de células semelhantes a fibroblastos. Ocasionalmente havia mineralização e proliferação na túnica íntima. Nas fibras musculares cardíacas havia irregularidade, fragmentação das fibras e deposição de grânulos basofílicos (mineralização) intracitoplasmáticos. No pulmão havia variável mineralização e deposição de material eosinofílico similar a colágeno em septos alveolares. Notou-se também mineralização em forma de placas nas cartilagens bronquiais e ocasionalmente formação de trabéculas ósseas (Fig.6).

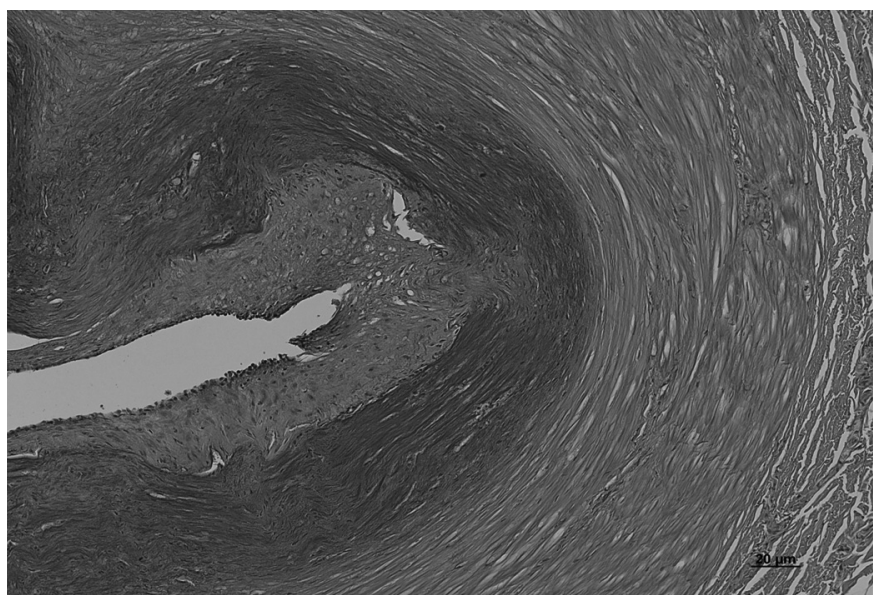

Fig.5. Carótida de búfalo exibindo formação de placas basofílicas, irregulares, multifocais por vezes coalescente principalmente na túnica média. Há também desorganização e fragmentação das fibras na túnica média. Intoxicação natural por Solanum glaucophyllum. HE, obj.10x.

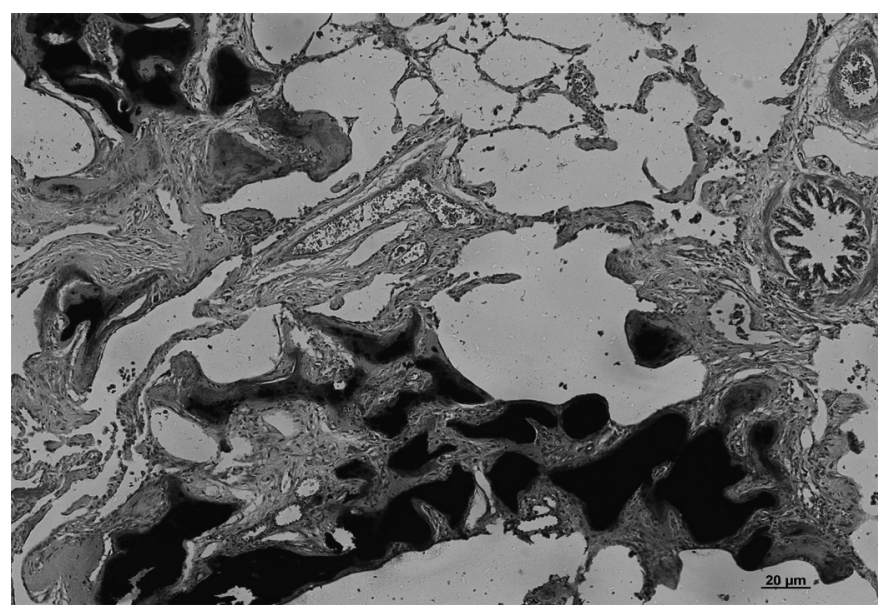

Fig.6. Pulmão de búfalo com variável mineralização em septos alveolares, proliferação de tecido conjuntivo, deposição de colágeno e enfisema alveolar. Há também mineralização em forma de placas nas cartilagens bronquiais e ocasionalmente metaplasia óssea. Intoxicação natural por Solanum glaucophyllum. Von Kossa, obj.10x.

\section{DISCUSSÃO}

Os achados epidemiológicos, clínicos e patológicos foram consistentes e compatíveis com o diagnóstico de intoxicação natural por Solanum glaucophyllum. Evidências adicionais foram obtidas com exames ultrassonográficos.
As particularidades comportamentais da espécie bufalina podem ter influenciado na dinâmica da enfermidade. A ocorrência dos casos no período chuvoso pode estar associada ao costume dos bufalinos de se alimentar, de forma menos seletiva, de plantas parcial ou totalmente submersas em alagados (Ford 1992, Vale 1999). No entanto, não podemos descartar que a permanência do rebanho em áreas onde há $S$. glaucophyllum no período da seca, pode ter levado ao consumo direto, ou mesmo indireto da planta desfolhada. Os quadros naturais descritos em bovinos ocorrem durante o período de estiagem no pantanal devido ingestão de folhas secas junto com forragem, uma vez que nesta espécie não há o consumo direto dos arbustos (Tokarnia \& Döbereiner 1974). Não foram observados bovinos com quadro clínico de calcinose, na inspeção visual, na propriedade onde os búfalos adoeceram. Porém, estes não permaneciam por longos períodos nas mesmas áreas que os búfalos, devido excesso de umidade e alagamento. Ressalte-se que os bovinos preferem locais mais altos e secos, denominados cordilheiras, para repouso e pastoreio. Os búfalos, pela intolerância à radiação solar direta, preferem notadamente a água de imersão como mecanismo de termólise, sendo recurso de proteção para dias quentes (Ablas et al. 2007), praticamente uma constante climática na região do pantanal. Este comportamento dos bufalinos, aliado ao habitat palustre da S. glaucophyllum, pode ter sido um fator predisponente para que ocorresse a intoxicação.

Caquexia progressiva, cifose (dorso arqueado), membros torácicos ligeiramente fletidos e apoio sobre as pinças, observados nos búfalos são semelhantes aos sinais relatados em casos de calcinose enzoótica em outros ruminantes (Döbereiner et al. 1971, Tokarnia \& Döbereiner 1974, Riet-Correa et al. 1993, Arnold \& Fincham 1997, Braun et al. 2000a,b, Franz et al. 2007, Rissi et al. 2007). Pelo que se observou no rebanho bufalino, a disfunção dos membros torácicos, são verificadas apenas na fase final de evolução, ou seja, quando os animais exibem sinais extremos de caquexia.

De acordo com Tokarnia (2009) animais que desenvolvem calcinose apresentam andar com o dorso arqueado e o metacarpo por vezes flexionado, fato que pode ser interpretado como uma postura antiálgica, e ao que parece, a hipótese é consistente, pois os achados envolvendo microcalcificações tendíneas relacionam-se aos animais mais gravemente afetados, que sendo observados a campo, pastam muito tempo ajoelhados (posição carpeada) e quando se levantavam exibiam dificuldades locomotoras, tais como claudicações e andar rígido. Diversas causas podem levar a dor ou disfunção de membros e dentre elas, a tendinose calcificante é apontada como causa em caninos e humanos (Muir \& Johnson 1994, Beale 2002). Ainda, nos achados ultrassonográficos foram visibilizadas inúmeras microcalcificações focais no tendão do músculo flexor digital profundo acima e abaixo dos sesamóides proximais. Embora ainda não descrito em búfalos, estes achados foram relatados em bovinos nos quais ocorrem macroscopicamente calcificações nos tendões sob a forma de pontilhados brancos na periferia e ligamentos dos sesamóides na superfície de corte com intensidades variáveis. Em condições experimentais 
calcificação nesta área apareceu em poucos animais e provavelmente, este achado pode relacionar-se a pequenas e constantes doses ingeridas de S. glaucophyllum diariamente, em casos naturais por longos períodos (Döbereiner et al. 1971). Franz et al. (2007) demonstraram que a ultrassonografia é mais uma ferramenta auxiliar no diagnóstico de calcinose, embora não tenha avaliado tendões em estudo experimental, detectou calcificações em outros pontos como aorta, válvulas cardíacas e rins de bovinos e ovinos.

Um fato importante de se ressaltar é que os dois búfalos mais gravemente afetados que não reverteram sinais, estavam recém paridas e apresentaram supressão da produção de leite, com morte dos neonatos após duas e quatro semanas de vida. A diminuição ou supressão láctea, já foi descrita em caprinos (Braun et al. 2000a) e bovinos (Braun et al. 2000b) em condições naturais, relacionadas a ingestão de Trisetum flavescens, planta que, também, apresenta atividade calcinogênica .

Outro sinal clínico observado foi a perda progressiva de pêlos e aparente hipotricose em dois búfalos deste estudo. Trabalhos anteriores descrevem alteração na pelagem, enfatizando pêlos ásperos em bovinos (Döbereiner et al. 1971, Tokarnia \& Döbereiner 1974) ou eriçados em ovinos (Franz et al. 2007). De acordo com Tonhati (2009) é comum perda parcial de pêlos nos búfalos em algumas épocas do ano, determinadas por condições de altas temperaturas. Pode-se pensar que animais em quadros avançados de calcinose poderiam exacerbar esta característica fisiológica pela ação do consumo da planta, e ainda, pelo maior tempo de permanência em decúbito dos animais intoxicados.

As calcificações de artérias, pulmões e tendões nos bufalinos, foram semelhantes aos descritos em bovinos (Döbereiner et al. 1971, Döbereiner \& Tokarnia 1974, Braun et al. 2000a,b, Tokarnia et al. 2000, Rissi et al. 2007), entretanto, calcificações renais que ocorrem na maioria dos bovinos com calcinose enzoótica (Tokarnia \& Döbereiner 1974) não foram verificadas nos búfalos.

Relatos de Tokarnia e Döbereiner que na década de 70 estudaram amplamente a dinâmica da doença em bovinos na região de Poconé-MT, apontam que a mudança dos animais para áreas que não exista a planta, permite recuperação parcial. Notou-se recuperação clínica aparente em dois búfalos deste estudo, no entanto outros dois que apresentaram sinais clínicos acentuados não se recuperaram e definharam. Análise comparativa entre estas duas espécies se faz necessário, como efetuado em outros estudos relativos a plantas e aditivos alimentares com potencial tóxico (Barbosa et al. 2003, Rozza et al. 2007), uma vez que diferenças quanto à resistência e susceptibidade podem ser marcantes entre estas espécies animais.

Agradecimentos.- À A.V. Thuronyi pela acessibilidade na área de estudo e ao Médico Veterinário C. Figueiredo pela cooperação nos trabalhos de campo. Agradecemos também aos Professores Carlos H. Tokarnia, H. Tonhati e J.J. Fagliari pelas informações e opiniões acerca do trabalho. À Coordenação de Aperfeiçoamento de Pessoal de Nível Superior (CAPES) pela concessão de bolsa do Programa Prodoutoral.

\section{REFERÊNCIAS}

Ablas D.S., Titto E.A.L., Pereira A.M.F., Titto C.G. \& Leme T.M.C. 2007. Comportamento de bubalinos a pasto frente a disponibilidade de sombra e água para imersão. Ciênc. Anim. Bras. 8:167-175.
Arnold R.M. \& Fincham I.H. 1997. Manchester wasting disease: A calcinosis caused by a pasture grass (Stenotaphrum secundatum) in Jamaica. Trop. Anim. Hlth Prod. 29:174-176.

Barros S.S., Driemeier D., Santos M.N. \& Guerreiro J.A.M. 1992. Evolução clínica e reversibilidade das lesões da calcinose enzoótica dos ovinos induzida por Nierembergia veitchii. Pesq. Vet. Bras. 12:5-10.

Barbosa J.D., Oliveira J.M.C., Tokarnia C.H. \& Riet-Correa F. 2003. Comparação da sensibilidade de bovinos e búfalos a intoxicação por Palicourea marcgravii (Rubiaceae). Pesq. Vet. Bras. 23:167-172.

Beale B. 2002. Supraspinatus tendon injuries. Proc. $1^{\text {st }}$ World Orthopaedic Veterinary Congress, Munich, p.51-52.

Boabaid F. M., Antoniassi N A.B., Bourscheid D., Soares L.C M., Santos C.E.P., Nakazato L. \& Colodel E.M. 2006. Aspectos clínicos e patológicos da calcinose enzoótica de etiologia desconhecida em rebanhos de ovinos do estado de Mato Grosso. Anais 33ํㅡㄹ Conbravet, Cuiabá, MT, p.230. (Resumo)

Braun U., Diener M., Camenzind D., Flückiger M. \& Thoma R. 2000a. Enzootic calcinosis in goats caused by golden oat grass (Trisetum flavescens). Vet. Rec. 146:161-162.

Braun U., Diener M., Hilbe M., Busch M., Bischoff M. \& Brosi G. 2000b. Enzootic calcinosis in 16 cows from 6 dairy farms in Unteregadin. Schweiz. Arch. Tierheilkd. 142:333-338.

Copland J.W. 1975. Enzootic calcinosis of cattle in Papua New Guinea. Aust. Vet. J. 51:326. (Apud Tokarnia et al. 2000)

Dirksen G., Plank P., Spiess A., Hänichen T. \& Dämmrich K. 1970. Über eine enzootische "Kalzinose" beim Rind. Dtsch. Tierärztl. Wschr. 77:321-346. (Apud Tokarnia et al. 2000)

Döbereiner J., Tokarnia C.H. , Costa J.B.D., Campos J.L.E. \& Dayrel M.S. 1971. "Espichamento", intoxicação de bovinos por Solanum malacoxylon, no Pantanal de Mato Grosso. Pesq. Agropec. Bras., Sér. Vet. 6:91-117.

Ford B.D. 1992. Swamp buffaloes in large scale ranching systems, p.465481. In: Tulloh N.M. \& Holmes J.H.G. (Eds), Buffalo Production. Elsevier, Amsterdam

Franz S., Gasteiner J., Schilcher F. \& Baumgartner W. 2007. Use of ultrasonography to detect calcifications in cattle and sheep fed Trisetum flavescens silage. Vet. Rec. 161:751-754.

Krook L., Wasserman R.E., McEntee K., Brokken T.D. \& Teigland M.D. 1975a. Cestrum diurnum poisoning in Florida cattle. Cornell Vet. 65:557575. (Apud Tokarnia et al. 2000)

Krook L., Wasserman R.H., Shively J.N., Tashjian A.H., Brokken T.D. \& Morton J.F. 1975b. Hypercalcemia and calcinosis in Florida horses: implication of the shrub Cestrum diurnum, as the causative agent. Cornell Vet. 65:26-56. (Apud Tokarnia et al. 2000)

Luna LG. 1968. Manual of histologic staining methods of the Armed Force Institute of Pathology. McGraw-Hill Book Company, New York, USA. 258p.

Mello J.R. B. 2003. Calcinosis: Calcinogenic plants. Toxicon 41:1-12.

Muir P. \& Johnson K.A. 1994. Supraspinatus and biceps brachii tendinopathy in dogs. J. Small Anim. Pract. 35:239-243.

Nicholson M.J. \& Butterworth M.H. 1986. A Guide to Condition Scoring of Zebu Cattle. International Livestock Centre for Africa, Addis Abeba. $29 \mathrm{p}$.

Riet-Correa F., Schild A.L., Méndez M.C., Wasserman R. \& Krook L. 1987. Enzootic calcinosis in sheep caused by the ingestion of Nierembergia veitchii (Solanaceae). Pesq. Vet. Bras. 7:85-95.

Riet-Correa F., Méndez M.C., Schild A.L. \& Petiz C.A. 1993. Enzootic calcinosis in sheep: Experimental reproduction with Nierembergia veitchii (Solanaceae). Pesq. Vet. Bras. 13:21-24.

Riet-Correa F., Schild A.L., Lemos R.A.A. \& Borges J.R.J. 2007. Intoxicação por plantas e micotoxinas, p.182-184. In: Ibid. (Eds), Doenças de Ruminantes e Equídeos. Vol.2. 3a ed. Editora Pallotti, Santa Maria.

Rissi D.R., Rech R.R., Pierezan F., Kommers G.D. \& Barros C.S.L. 2007. Intoxicação em ovinos por Nierembergia veitchii: observações em quatro surtos. Ciência Rural 37:1393-1398. 
Rozza D.B., Correa A.M. R., Leal J.S., Bandarra P.M., Guagnini F.S., Raymundo G.R. \& Driemeier D. 2007. Intoxicação experimental por monensina em búfalos e bovinos. Pesq. Vet. Bras. 27:172-178.

Santos A.S., Souza M.A., Brum K.B. \& Jorge P.H.C. 2005. Calcinose em ruminantes de etiologia desconhecida no Estado de Goiás. Anais XII Enapave, Belo Horizonte, MG, p.148. (Resumo)

Tokarnia C.H. 2009. Comunicação pessoal (Departamento de Nutrição Animal e Pastagem, Universidade Federal Rural do Rio de Janeiro, Seropédica, RJ).

Tokarnia C.H. \& Döbereiner J. 1974. “Espichamento”, intoxicação de bovi- nos por Solanum malacoxylon, no Pantanal de Mato Grosso. II. Estudos complementares. Pesq. Agropec. Bras. 9:53-62.

Tokarnia C.H., Döbereiner J. \& Peixoto P.V. 2000. Plantas que causam calcificação sistêmica (calcinose), p.189-199. In: Ibid. (Eds), Plantas Tóxicas do Brasil. Editora Helianthus, Rio de Janeiro.

Tonhati H. 2009. Comunicação pessoal (Departamento de Zootecnia, Universidade Estadual Paulista Júlio de Mesquita Filho, Jaboticabal, SP).

Vale W.G. 1999. Perspectivas da bubalinocultura no Brasil e na América Latina, p.1-26. In: Tonhati H., Barnabé V.H. \& Baruselli PS. (Eds), Bubalinos: sanidade, reprodução e produção. Funep, Jaboticabal. 